\title{
Incomplete Andreev reflection in a clean SFS junction
}

\author{
Jérôme Cayssol ${ }^{1,2}$ and Gilles Montambaux ${ }^{1}$ \\ (1)Laboratoire de Physique des Solides, Associé au CNRS, Université Paris Sud, 91405 Orsay, France and \\ (2)Laboratoire de Physique Théorique et Modèles Statistiques, \\ Associé au CNRS, Université Paris Sud, 91405 Orsay, France
}

\begin{abstract}
We study the Josephson effect in a clean Superconductor-Ferromagnet-Superconductor junction for arbitrarily large spin polarizations. The Andreev reflection at a clean FerromagnetSuperconductor interface is incomplete, and Andreev channels with a large incidence angle are progressively suppressed with increasing exchange energy. As a result, the critical current exhibits oscillations as a function of the exchange energy and of the length of the ferromagnet and has a temperature dependence which deviates from the one predicted by the quasiclassical theory.
\end{abstract}

Current understanding of the SuperconductorFerromagnet-Superconductor (SFS) Josephson effect is limited to small spin polarizations. In the case of conventional superconductors, the Josephson current is due to the Andreev [1] conversion of singlet Cooper pairs into correlated electrons and holes with opposite spins propagating coherently in the ferromagnetic metal. Applying the Eilenberger equations [2 to a clean multichannel SFS junction, Buzdin et al. [3] have predicted that this non dissipative current oscillates as a function of both the exchange energy splitting $E_{e x}$ and the length $d$ of the ferromagnet, because of the mismatch $2 E_{e x} / \hbar v_{F}$ between the spin-up and spin-down Fermi wavevectors. This quasiclassical result assumes that the Andreev reflection is complete, as it is fully justified for weakly spin-polarized ferromagnetic alloys $E_{e x} \ll E_{F}, E_{F}$ being the Fermi energy. First experimental evidence for such oscillating critical current has recently been reported in $\mathrm{Nb}-\mathrm{Cu}-\mathrm{Ni}-\mathrm{Cu}-\mathrm{Nb}$ junctions [4]. The so-called $\pi$-phase state of a SFS junction [5] has also been observed using diffusive weak ferromagnetic alloys such as $\mathrm{Cu}_{1-x} \mathrm{Ni}_{x} \quad[\underline{6}$ or $\mathrm{Pd}_{1-x} \mathrm{Ni}_{x}$ [7], [8],, 9$]$.

In the new field of spintronics, devices with high spin polarization are used in order to manipulate spin polarized currents. In the recently discovered half metals (HM), such as $\mathrm{CrO}_{2}$ and $\mathrm{La}_{0.7} \mathrm{Sr}_{0.3} \mathrm{MnO}_{3}$, the current is completely spin polarized because one spin subband is insulating. Ferromagnetic elements $\mathrm{Fe}, \mathrm{Co}, \mathrm{Ni}$, also exhibit quite large spin polarizations. Anticipating the interest for large spin polarizations, de Jong and Beenakker [10] have shown that in this case the Andreev reflection is not complete at a clean Ferromagnet-Superconductor (FS) interface, in contrast to the case of a clean nonmagnetic Normal metal-Superconductor (NS) interface. Even in the absence of impurity scattering, normal reflection may occur because of the diagonal exchange potential barrier between the ferromagnet and the superconductor. This suppression of the Andreev reflection affects preferentially the channels with large transverse momentum. As a result, the sub-gap conductance of a ballistic FS contact decreases quasi-linearly as a function of the spin polarization $\eta=E_{e x} / E_{F}$ from twice the normal state conduc- tance $(\eta=0)$ to zero $(\eta=1)$, because of the progressive suppression of the Andreev process. Using this principle, a point-contact Andreev reflection technique has been developed in order to mesure directly the spin polarization of materials [1], 12], such as $\mathrm{La}_{0.7} \mathrm{Sr}_{0.3} \mathrm{MnO}_{3}, \mathrm{CrO}_{2}$, $\mathrm{NiFe}, \mathrm{NiMnSb}$, which were not easily accessible by spin resolved tunneling spectroscopy [13]. A huge amount of theoretical efforts has been devoted to transport properties in a nanoscale FS contact [14, [15], 16], 17] while few studies have considered the thermodynamical properties of FS heterostructures [18], 19].

In this Letter, we address the physics of the Josephson effect in a clean multichannel SFS junction in the range of arbitrarily large spin polarization. We show how the Josephson current is modified by the ordinary reflection induced by the ferromagnet in the crossover from a SNS $(\eta=0)$ to a $\mathrm{S} / \mathrm{HM} / \mathrm{S}$ junction $(\eta>1)$. With increasing exchange energy, the Andreev reflection is suppressed for electrons propagating with a large incidence, so that the number of channels contributing to the total current decreases. This reduction of the number of "Andreev active channels" has furthermore a subtle effect on the Josephson current: although the FS conductance is always reduced when $\eta$ increases [10], the critical current has a non-monotonic behavior, depending on the currentphase relationship of the suppressed channels. For large spin polarizations, the oscillations of the critical current depend separately on the product $k_{F} d$ and on the spin polarization $\eta$. They are reduced and shifted with respect to the predictions of the quasiclassical theory [3] in which only a single parameter, $2 E_{e x} d /\left(\hbar v_{F}\right)=\eta k_{F} d$, is relevant. For small spin polarizations, we naturally recover the quasiclassical results. In the HM limit $E_{e x} \rightarrow E_{F}$, the critical current vanishes because the Andreev reflection is totally suppressed for all the transverse channels. In addition, we study the temperature dependence of the critical current for different values of the spin polarization and of the length $d$ of the ferromagnet.

We consider a clean short SFS junction with a large number $M$ of transverse channels and with a length $d$ of the ferromagnetic region much smaller than the coherence length of the superconductor $\xi_{o}=\hbar v_{F} / \Delta_{o}$, where 
$\Delta_{o}$ is the $T=0$ superconducting gap. The itinerant ferromagnetism is described within the Stoner model by an effective one body potential $V_{\sigma}(x)=-\sigma E_{e x}$ which depends on the spin direction, characterized by $\sigma= \pm 1$. In the superconducting leads, $V_{\sigma}(x)=0$. The superconducting pair potential is $\Delta(x)=|\Delta| e^{i \chi / 2}$ in the left lead and $\Delta(x)=|\Delta| e^{-i \chi / 2}$ in the right lead. In the absence of spin-flip scattering, the Bogoliubov-de Gennes equations split in two sets of independent equations for the spin channels $\left(u_{\uparrow}, v_{\downarrow}\right)$ and $\left(u_{\downarrow}, v_{\uparrow}\right)$

$$
\left(\begin{array}{cc}
H_{o}+V_{\sigma}(x) & \Delta(x) \\
\Delta(x)^{*} & -H_{o}^{*}+V_{\sigma}(x)
\end{array}\right)\left(\begin{array}{l}
u_{\sigma} \\
v_{-\sigma}
\end{array}\right)=\epsilon(\chi)\left(\begin{array}{l}
u_{\sigma} \\
v_{-\sigma}
\end{array}\right),
$$

where $\epsilon(\chi)$ is the quasiparticle energy mesured from the Fermi energy 20]. The kinetic part of the Hamiltonian $H_{o}=\left[(-i \hbar d / d x-q A(x))^{2}-E_{F}\right] / 2 m$, with the effective mass of electron and hole $m$, is expressed in terms of the vector potential $A(x)$, which is responsible for the phase difference $\chi$ between the leads, and $E_{F}=\hbar^{2} k_{F}^{2} / 2 m$ is the Fermi energy. The Fermi velocities are identical in both superconductors and in the paramagnetic metal.

Because both the pair and the disorder potential are identically zero in the ferromagnet, the eigenvectors of Eq. (1) are electrons and holes with plane wave spatial dependencies. For a given transverse channel, the electron and hole longitudinal wavevectors, $k_{n \sigma}$ and $h_{n-\sigma}$ respectively, satisfy

$$
\begin{aligned}
\frac{\hbar^{2} k_{n \sigma}^{2}}{2 m}+E_{n} & =E_{F}+\epsilon+\sigma E_{e x}, \\
\frac{\hbar^{2} h_{n-\sigma}^{2}}{2 m}+E_{n} & =E_{F}-\epsilon-\sigma E_{e x},
\end{aligned}
$$

where $E_{n}$ is the transverse energy of the channel. One may label the transverse channels by an angle $\theta_{n}$ which is the incidence angle of the corresponding quasiparticle trajectory

$$
E_{n}=\frac{\hbar^{2} k_{F}^{2}}{2 m} \sin ^{2} \theta_{n}=E_{F} \sin ^{2} \theta_{n}
$$

From Eq. (2), one sees that an electron with incidence $\theta_{n}$ cannot form an Andreev bound state with a hole if $E_{n}=E_{F} \sin ^{2} \theta_{n}>E_{F}-E_{e x}$. Therefore the electron is normally reflected as an electron with the same spin for angle $\theta_{n}>\theta_{\eta}=\arccos \sqrt{\eta}$. Such a process is insensitive to the superconducting phase and thus carries no Josephson current. In the opposite case $\theta_{n}<<\theta_{\eta}$, the Andreev reflection is complete and supports a finite current. In the following, the former kind of channel is referred to as "Andreev inactive" and the latter as "Andreev active".

Recently, we have performed detailed studies of the spectrum of a single channel SFS junction for arbitrarily large exchange energies 21. Solving the Bogoliubov-de Gennes equations, the spectrum is found to be strongly modified in comparison to the quasiclassical spectrum 22. because gaps open at $\chi=0$ and $\chi=\pi$. However, due to a cancellation between the corrections associated to each anticrossing, the current is almost unaffected up to very large spin polarizations $\eta \approx 0.95$. The region in which Andreev reflection and ordinary reflection coexist is extremely small. As a result, the Josephson current through a single channel SFS junction is given to great accuracy by the formula for perfect Andreev reflection [3]

$$
\begin{aligned}
i\left(\chi, k_{F} d, \eta, \theta_{n}=0\right)= & \frac{\pi \Delta}{\phi_{o}} \sum_{\sigma= \pm 1} \sin \frac{\chi+\sigma a}{2} \\
& \tanh \left(\frac{\Delta}{2 T} \cos \left(\frac{\chi+\sigma a}{2}\right)\right),
\end{aligned}
$$

for $\eta<1$ and it is zero for $\eta>1$. The parameter $a=$ $(\sqrt{1+\eta}-\sqrt{1-\eta}) k_{F} d$ is the phase shift accumulated between an electron and a hole located at the Fermi level during their propagation on a length $d$.

In the present paper, we generalize this result to transverse channels with finite angle $\theta_{n}$, in the more realistic case of a finite width SFS junction. The crossover between Andreev active and inactive channels occurs in a narrow window of incidences in the vicinity of $\theta_{\eta}=$ $\arccos \sqrt{\eta}$. Below this cut-off, the current carried by a single Andreev active channel is

$$
\begin{aligned}
i\left(\chi, k_{F} d, \eta, \theta_{n}\right)= & \frac{\pi \Delta}{\phi_{o}} \sum_{\sigma= \pm 1} \sin \frac{\chi+\sigma a_{n}}{2} \\
& \tanh \left(\frac{\Delta}{2 T} \cos \left(\frac{\chi+\sigma a_{n}}{2}\right)\right),
\end{aligned}
$$

and it is zero for $\theta_{\eta}>\arccos \sqrt{\eta}$. In order to treat large exchange splitting, one has to take into account the exact band structure (here a simple isotropic parabolic band) and to express the phase shift between an electron and its Andreev reflected hole by

$$
a_{n}=k_{F} d \cos \theta_{n}\left(\sqrt{1+\frac{\eta}{\cos ^{2} \theta_{n}}}-\sqrt{1-\frac{\eta}{\cos ^{2} \theta_{n}}}\right),
$$

instead of using the linearized form

$$
a_{n}=\frac{\eta k_{F} d}{\cos \theta_{n}}=\frac{2 E_{e x} d}{\hbar v_{F} \cos \theta_{n}} .
$$

The transverse channels considered above are independent because $V_{\sigma}(x)$ is translationaly invariant in the transverse directions. Thus, the total current is the sum of the currents carried by each of them. As we assume a large number of channels, the discrete sum over $n$ can be replaced by an integral over the angle $\theta$. Calculating the total current, one has to restrict the integration over Andreev active levels only, so that the angular integral has to be limited by the upper cut-off $\theta_{\eta}=\arccos \sqrt{\eta}$

$$
I\left(\chi, k_{F} d, \eta\right)=\frac{k_{F}^{2} S}{2 \pi} \int_{0}^{\theta_{\eta}} d \theta \sin \theta \cos \theta i\left(\chi, k_{F} d, \eta, \theta\right),
$$






FIG. 1: Current-phase relationships at zero temperature for $a=\pi / 4$ obtained for several pairs $\left(\eta, k_{F} d\right)$. In the quasiclassical approximation, the current is a function of the single parameter $a$ and does not decrease with increasing $\eta$. The current is given in units of $I_{o}=\pi \Delta_{0} /\left(e R_{N}\right)$

where $S$ is the cross section area of the ferromagnet.

This expression, together with Eqs. (5) [6), is the central result of this Letter. It gives the Josephson current $I\left(\chi, k_{F} d, \eta\right)$ of a clean SFS junction in the regime of arbitrarily large spin polarization. Examples of current-phase relationships are shown in Fig. 1] In the limit of small polarization $\eta=E_{e x} / E_{F} \rightarrow 0$, we recover the quasiclassical current-phase relationship [3] in which all the transverse channels contribute because $\theta_{\eta} \rightarrow \pi / 2$.

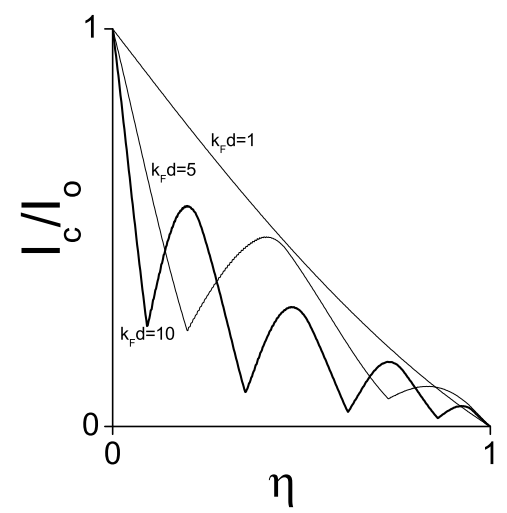

FIG. 2: Zero temperature critical current $I_{c}(\eta)$ as a function of $\eta=E_{e x} / E_{F}$ for different lengths of the ferromagnet, $k_{F} d=$ $1,5,10$. The current is given in units of $I_{o}=\pi \Delta_{0} /\left(e R_{N}\right)$.

Increasing the spin polarization, we study how the critical current evolves from the case of a weakly spin polarized junction to the S/HM/S junction. As shown in Fig. 2 the critical current has a non trivial oscillatory behavior as a function of exchange splitting for a given length, namely for fixed $k_{F} d$. The number of oscillations occuring during the crossover from the SNS $(\eta=0)$ to the $\mathrm{S} / \mathrm{HM} / \mathrm{S}$ junction $(\eta=1)$ decreases when $k_{F} d$ is lowered. In the limit of an ultra-small junction $k_{F} d \approx 1$, there are no oscillations because the phase shift in Eq. (6) tends to zero, and all transverse channels carry the same SNS current with maximal value $i_{o}=2 \pi \Delta / \phi_{0}$, where $\phi_{o}=h / e$
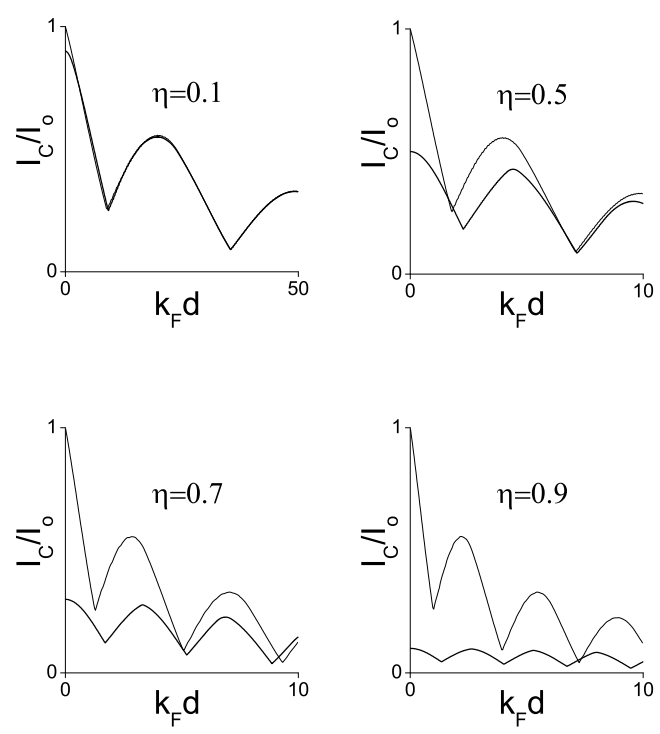

FIG. 3: Zero temperature critical current $I_{c}(\eta)$ as a function of $k_{F} d$ (thick lines), for different values of the spin polarization $\eta$. As $\eta$ increases, the exact current deviates from the quasiclassical estimate (dashed lines). The current is given in units of $I_{o}=\pi \Delta /\left(e R_{N}\right)$.

is the flux quantum. Consequently, the reduction of the total current is only governed by the upper cut-off in Eq. (8)

$$
I_{c}=M i_{0}(1-\eta)=\frac{\pi \Delta}{e R_{N}}(1-\eta)
$$

This linear reduction of the current with increasing the exchange field is quite reminiscent of the almost linear reduction obtained in Ref. 10] for the conductance of a FS nanocontact 23]. The total number of transport channels $M=k_{F}^{2} S / 4 \pi$ is large and determines the small normal state resistance $R_{N}=h /\left(2 e^{2} M\right)$ of the heterojunction. The natural unit for the critical current is $I_{o}=\pi \Delta / e R_{N}$, namely the one of a short clean SNS junction.

Fig. 3 represents the critical current as a function of the length $d$ of the ferromagnetic region, for different spin polarizations. We find that the oscillations are reduced and shifted with respect to the quasiclassical calculation. There are two reasons for these deviations. Firstly, trajectories with large incidence are progressively suppressed. Secondly, the phase shift between electrons and holes for a given channel [Eq. [6] ] depends on the particular band structure and differs from the linearized version $a_{n}=\eta k_{F} d / \cos \theta_{n}$. For large $d$, the oscillations decay slowly at zero temperature. In real situations, they are expected to be severely reduced when $d$ exceeds the thermal length $L_{T}=\hbar v_{F} / T$ or the phase coherence length $L_{\phi}(T)$.

We finally consider the effect of a finite temperature on the critical current. We have adopted the BCS temperature dependence of the order parameter $\Delta(T)=$ 


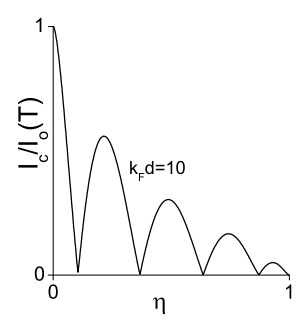

(a)

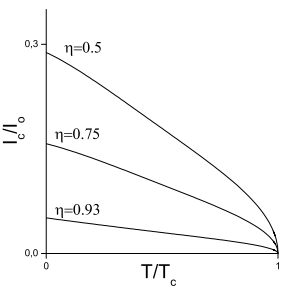

(b)

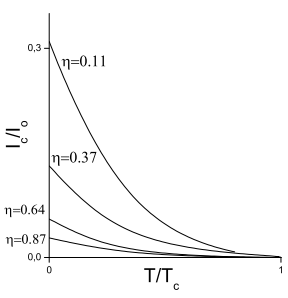

$(c)$
FIG. 4: a) Critical current as a function of the spin polarization $\eta$ at $T=0.9 T_{c}$. It vanishes for particular values of the spin polarization, when the junction undergoes a $0-\pi$ transition. $I_{0}(T)=\pi \Delta(T)^{2} /\left(4 e R_{N} T_{c}\right)$ is the critical current for a SNS junction. b) Critical current [in units of $\left.I_{0}=\pi \Delta_{o} /\left(e R_{N}\right)\right]$ as a function of the reduced temperature $T / T_{c}$ for values of $\eta$ corresponding to the maxima of Fig (a). c) Critical current as a function of $T / T_{c}$ for different values of $\eta$ corresponding to the $0-\pi$ transitions. All curves correspond to a short junction with $k_{F} d=10$.

$\Delta_{o} \tanh \left(1.74 \sqrt{T_{c} / T-1}\right)$, and the exchange energy is assumed to be temperature independent. For $T \approx T_{c}$, Fig. 4(a) shows that the critical current oscillates with the spin polarization $\eta$ and cancels out for some values of $\eta$. In this temperature range, the current-phase relationship is sinusoidal $I(\chi)=I_{c} \sin \chi$ and the current vanishes identically when $I_{c}$ is zero. These cancellations are associated to transitions between the 0 -phase state and the $\pi$-state of the junction. For fixed parameters $k_{F} d$ and $\eta$, the critical current decreases monotonously with increasing temperature $T$, as shown in Figs. 4(b,c). This temperature dependence is very sensitive to the spin polarization. For polarizations corresponding to $0-\pi$ transitions, $I_{c}(T)$ decreases exponentially with temperature [Fig. 4(c)] whereas a much more slower decrease is obtained for the local maxima of the critical current [Fig. $4(\mathrm{~b})]$.

We have studied the Josephson current of a clean SFS junction for arbitrary large spin polarizations. The two physical effects involved are the reduction of the number of active levels participating in the Andreev process and the use of the non-linearized band structure. In any experiment with strong ferromagnetic elements or nearly half metallic compounds, the critical current oscillations should be affected by these effects. Firstly, the oscilla- tions depend separately on the spin polarization $\eta$ and on the product $k_{F} d$ instead of the single combination $\eta k_{F} d$ as suggested by the quasiclassical theory. Secondly, when the temperature is increased from zero to the critical temperature, the local minima of the current are more strongly suppressed than the local maxima.

We thank Igor Zutic for useful comments.

[1] A. F. Andreev, Sov. Phys. JETP 19, 1228 (1964).

[2] G. Eilenberger, Z. Phys. 214, 195 (1968).

[3] A.I. Buzdin, L.N. Bulaevskii, and S.V. Panyukov, JETP Lett. 35, 178 (1982).

[4] Y. Blum, A. Tsukernik, M. Karpovski, and A. Palevski, Phys. Rev. Lett. 89, 187004 (2002).

[5] L.N. Bulaevskii, V. V. Kuzii, and A.A. Sobyanin, Sov. Phys. JETP Lett. 25, 290 (1977).

[6] V.V. Ryazanov, V.A. Oboznov, A.Yu. Rusanov, A.V. Veretennikov, A.A. Golubov, and J. Aarts, Phys. Rev. Lett. 86, 2427 (2001).

[7] T. Kontos, M. Aprili, J. Lesueur, and X. Grison, Phys. Rev. Lett. 86, 304 (2001).

[8] W. Guichard, M. Aprili, O. Bourgeois, T. Kontos, J. Lesueur, and P. Gandit, Phys. Rev. Lett. 90, 167001 (2003).

[9] A. Bauer, J. Bentner, M. Aprili, M.L.D. Rocca, M. Reinwald, W. Wegscheider, and C. Strunk, cond-mat/0312165 (2003).

[10] M.J.M de Jong and C.W.J. Beenakker, Phys. Rev. Lett. 74, 1657 (1995).

[11] R.J. Soulen, Jr.J.M. Byers, M.S. Osofsky, B. Nadgorny, T. Ambrose, S.F. Cheng, P.R. Broussard, C. T. Tanaka, J. Nowak, J.S. Moodera, A. Barry, and J.M.D. Coey, Science 282, 85 (1998).

[12] S.K. Upadhyay, A. Palanisami, R.N. Louie, and R.A. Buhrman, Phys. Rev. Lett. 81, 3247 (1998).

[13] P.M. Tedrow and R. Meservey, Phys. Rev. B. 7, 318 (1973).

[14] I. Zutic and S. Das Sarma, Phys. Rev. B. 60, R16322 (1999).

[15] I. Zutic and O.T. Valls, Phys. Rev. B. 61, 1555 (2000).

[16] I.I. Mazin , Phys. Rev. Lett. 83, 1427 (1999).

[17] J. Kopu, M. Eschrig, J.C. Cuevas, and M. Fogelström, Phys. Rev. B. 69, 094501 (2004).

[18] K. Halterman and O.T. Valls, Phys. Rev. B. 65, 014509 (2001); cond-mat/0404058

[19] M. Eschrig, J. Kopu, J.C. Cuevas, and G. Schön, Phys. Rev. Lett. 90, 137003 (2003).

[20] P. G. de Gennes, Superconductivity of Metals and Alloys (Benjamin, New-York, 1966).

[21] J. Cayssol and G. Montambaux, cond-mat/0404190

[22] S.V. Kuplevakhskii and I.I. Fal'ko, JETP Lett. 52, 343 (1990).

[23] For a two-dimensional junction and in the limit $k_{F} d \ll 1$, the reduction of the critical current with the exchange field is given by $I_{c}=M i_{0} \sqrt{1-\eta}=\frac{\pi \Delta}{e R_{N}} \sqrt{1-\eta}$, where $M$ is the number of transverse channels in a two-dimensional stripe. 